\title{
Percepção do tempo: da psicologia para a psicolinguística
}

\author{
The perception of time: from Psychology to Psycholinguistics
}

Thiago Oliveira da Motta Sampaio ${ }^{1}$

Universidade Estadual de Campinas - Campinas - São Paulo - Brasil

$-\diamond$

\begin{abstract}
Resumo: As pesquisas sobre a percepção do tempo surgiram na psicologia com o objetivo de compreender os mecanismos envolvidos na cronobiologia, na estimação do tempo subjetivo e na coordenação temporal de algumas respostas motoras. Os avanços alcançados nesta disciplina resultaram em um modelo de processamento de informação que visa explicar nossa estimação do tempo subjetivo. Os estudos da linguagem lidam frequentemente com fenômenos de ordem temporal, como a coerção aspectual. A coerção ocorre quando um verbo pontual é inserido em contextos durativos, forçando uma leitura iterativa do evento [a criança espirrou o tempo todo]. Apenas recentemente, porém, o conhecimento sobre a percepção do tempo alcançaria a linguística, expandindo as previsões do fenômeno da coerção aspectual. Este trabalho consiste em uma breve revisão dos principais conceitos em percepção do tempo, buscando relaciona-los com as atuais hipóteses sobre coerção.
\end{abstract}

Palavras-chave: Percepção do tempo; Psicologia cognitiva; Psicolinguística; Coerção aspectual

\begin{abstract}
Research on time perception born aiming at understanding the mechanisms underlying chronobiology, the estimation of subjective time and the temporal coordination of some motor responses. The progress made by the discipline raised an information processing model that explains the cognitive mechanisms involved in the psychological time. Linguistic research also deals with some time-related phenomena, such as aspectual coercion. Coercion occurs when a punctual verb is inserted into durative contexts, forcing an iterative reading of the event [the child sneezed all the day long]. Only recently the concepts of subjective time reached the research on language and expanded the predictions of some linguistic phenomena. The present paper consists of a brief review of the main concepts on time perception, aiming at stablishing a relation between the psychology of time and the psycholinguistics of aspectual coercion.
\end{abstract}

Keywords: Time perception; Cognitive psychology; Psycholinguistics; Aspectual coercion

\section{Introdução}

Nos últimos quinze anos, os estudos em Linguística Experimental observaram um efeito que acontece em sentenças que combinam verbos pontuais e contextos durativos (ex. a criança pulou por alguns minutos). Este efeito pode ser encontrado através de experimentos de leitura automonitorada (TODOROVA et al. 2000a,b; BRENNAN, PYLKKÄNNEN, 2008; CHAN 2013), de rastreamento ocular da leitura (PICKERING 2006; TOWNSEND, 2012) e também através de métodos neurofisiológicos como eletroencefalografia (PACZYNSKI,

\footnotetext{
Este artigo é baseado no capítulo 4 de minha tese de doutorado. Uma versão prévia também foi apresentada no congresso Scientiarum Historia VIII na UFRJ.
}

KUPERBERG, 2014; YANO, 2016; BŁASZCZAK, KLIMEK-JANKOWSKA, a aparecer) e magnetoencefalografia (BRENNAN, PYLKKÄNEN, 2008). Visto que as propriedades em conflito no processamento deste tipo de sentença são conhecidas dos estudos do aspecto lexical (aktionsart) - a pontualidade do evento [pular] e a duratividade do contexto [por alguns minutos] - o fenômeno foi batizado com o nome Coerção Aspectual (PUSTEJOVSKY, 1995; JACKENDOFF, 1997).

Nos últimos anos, duas hipóteses alternativas foram propostas. Primeiramente, Dölling (2014) propõe que este efeito é, na verdade, devido a operação de mudança de classe dos verbos, segundo sua própria classificação de eventos. Desta forma, existiriam pelo menos nove tipos diferentes de coerção aspectual, todas elas operadas 
na Pragmática. Já Sampaio, França e Maia (2014) e SAMPAIO (2015), propõem que os efeitos encontrados nos estudos psicolinguísticos da coerção aspectual se devem a uma incompatibilidade semântica da duração subjetiva estimada para o evento em relação a duração de seu contexto. Esta visão abre a possibilidade para que verbos durativos também sejam afetados pelo mesmo fenômeno (ex. João cantou por algumas horas). Sua proposta se apoia em conhecimentos adquiridos pela psicologia na área da Percepção do Tempo.

O objetivo deste artigo é revisar os conhecimentos adquiridos sobre a percepção do tempo na psicologia experimental buscando discutir como se daria uma hipotética interface deste sistema com o processamento de sentenças. Neste trabalho darei foco ao fenômeno da coerção aspectual, apontando possíveis formas de avançar nesta área de pesquisa. Para isso, introduzo alguns conceitos de integração multissensorial, área da psicologia cognitiva que pesquisa a influência de diferentes modalidades de estímulos na percepção de um único evento no mundo. A integração multissensorial depende da percepção do tempo, tema a ser revisado em seus conceitos mais básicos nas seções 2 e 3. As seções 4 e 5 discutem as formas como os estudos da linguagem lidam com o conceito de tempo e da duração de eventos. Em seguida revisaremos a proposta de interface dos sistemas de percepção temporal e da linguagem de Sampaio, França e Maia (2014), finalizando com algumas considerações finais na seção 7 .

\section{Sobre a integração das informações sensoriais}

Quando estudamos sistemas sensoriais, a tendência é explicar os mecanismos físicos e sensitivos de forma individual como, por exemplo, a psicologia da visão ou a neurofisiologia da audição. Embora didática, esta divisão pode trazer algumas consequências indesejáveis nos leitores de primeira viagem.

$\mathrm{O}$ trabalho mais antigo conhecido que sugeria uma interação entre modalidades sensoriais data do final do século XIX, quando Urbantschitsch (1888, apud STEIN, 2012) evidenciou que indivíduos com danos cerebrais tinham um melhor desempenho em tarefas visuais quando o estímulo visual era acompanhado de estimulação auditiva. Desde então, alguns trabalhos replicaram seus achados e desenvolveram a disciplina. Ainda assim, estudos da interação entre modalidades sensoriais só começou a crescer nos anos 1980, quando pesquisadores tentaram compreender os efeitos intermodais no nível neuronal, dando força ao nascimento da Integração Multissensorial como disciplina na década de 90 (STEIN, 2012).
Um exemplo conhecido dos estudos da linguagem é a interação de estímulos audiovisuais, explorada por McGurk e MacDonald (1976). O Efeito McGurk se trata de uma ilusão perceptual que ocorre quando temos um desencontro entre as informações auditiva e visual. $\mathrm{O}$ experimento consiste na gravação de um vídeo em que uma pessoa pronuncia uma sílaba qualquer e de um áudio em que esta pessoa pronuncia uma segunda sílaba. Ao sincronizar o vídeo da sílaba 1 com o áudio da sílaba 2 , o participante tende a perceber uma terceira sílaba cujas propriedades se encontram entre as duas sílabas apresentadas (Fig. 1). Isso pode explicar o porquê de nos sentirmos tão desconfortáveis ao assistir filmes com dublagem ruim.

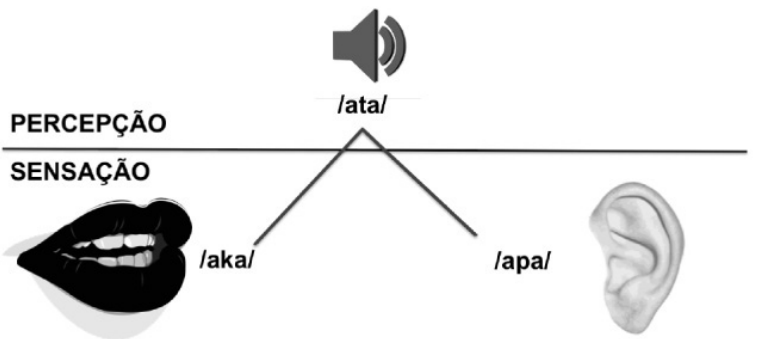

Figura 1. Ao estimular um voluntário visualmente com o os movimentos labiais que gerariam a sensação do som [aka] e o estímulo auditivo que geraria a sensação fonética do som [apa], no nível fonológico, os voluntários teriam a percepção fonológica do som /ata/ cuja consoante /t/ é alveolar, se encontrando em posição intermediária entre a consoante velar [k] e a consoante labial [p].

Um ponto importante a ser discutido sobre as razões para a interferência de uma modalidade em outra é o mecanismo que permite que esta integração aconteça. Voltando para o efeito McGurk, sabemos os estímulos visuais e auditivos chegam aos nossos neurônios sensitivos em velocidades diferentes. A luz se propaga próximo a $300.000 \mathrm{~km} / \mathrm{s}$. O som, por outro lado, se propaga a $340 \mathrm{~m} / \mathrm{s}$ no nível do mar, o que corresponde a uma velocidade 880 mil vezes mais lenta que o estímulo visual. Além disso, os mecanismos de processamento também são diferentes. O som é processado no córtex auditivo no lobo temporal, enquanto o estímulo visual será processado pelo córtex visual no lobo occipital. Levando em consideração que os processos de percepção costumam ser medidos na escala dos milissegundos e que a velocidade de transmissão do sinal pelos neurônios também é limitada, girando em torno de $100 \mathrm{~m} / \mathrm{s}$, parece improvável que o processamento das duas modalidades seja perfeitamente simultâneo.

Em resumo, a integração multissensorial busca responder a esta pergunta: como podemos perceber estímulos de natureza, de velocidade e de processamento tão distintos como se fossem simultâneos e correspondentes 
ao mesmo evento? A proposta dos pesquisadores da área indica que o cérebro não processa todas as informações assim que elas chegam. Ele aguarda algum tempo para poder integrar as informações. Diversos cientistas buscaram calcular este tempo de espera, que parece estar próximo de 100ms (STETSON et al., 2006; HAGGARD, 2008; ZHAO et al., 2013). Apesar de positivo, este atraso pode trazer consequências indesejadas para a percepção do estímulo, como percebemos em ilusões temporais como a Stopped Clock Illusion (GEORG, LAPPE, 2007), a Flash Lag Illusion (EAGLEMAN, SEJNOWSKI, 2007), e a Cronostase (VAN WASSENHOVE et al., 2008).

Como a integração multissensorial busca pelo tempo de integração dos estímulos no cérebro, esta disciplina faz interface direta com os estudos da Percepção do Tempo.

\section{Conceitos básicos sobre Percepção do Tempo}

O tempo não é uma variação de energia que possa estimular os neurônios sensitivos. Ao contrário da visão, não existem organismos que possuam sensores especializados em captar informações temporais do mundo externo. Apesar disso, a noção de tempo parece ser crucial em nossas vidas e em nosso comportamento.

$\mathrm{Na}$ tentativa de decifrar os mecanismos que nos permitem perceber e monitorar a passagem do tempo, as ciências cognitivas dividem o conceito em três partes. $\mathrm{O}$ 〈tempo objetivo〉 se refere ao tempo enquanto medida, que pode ser contado no relógio. Quando vemos um trovão rasgar os céus, podemos medir o tempo necessário para que as informações visual e auditiva cheguem até nossas retinas e ouvidos. Estas informações, porém, viajam em velocidades distintas, chegam aos nossos órgãos perceptuais em momentos distintos e são processadas em locais diferentes do cérebro. Isso indica que o tratamento das informações sensoriais não acontece de forma linear, do contrário seríamos incapazes de atribuir imagem e som ao mesmo estímulo (Integração Multissensorial). Estudos em percepção do tempo automático apontam que o cérebro aguarda e forma blocos de informações que serão tratados para então construir a percepção do 'Tempo Implícito' (STETSON et al., 2006²; PARIYADATH, EAGLEMAN, 2007). Após o processamento, o resultado será a nossa percepção subjetiva do tempo decorrido, o que chamamos de 'Tempo Explícito' ou 'Tempo Subjetivo'.

Para medir a relação entre tempo objetivo e subjetivo (Ponto de Simultaneidade Subjetiva - PSS) é

\footnotetext{
2 Stetson et al (2006), em especial, consegue reverter a percepção de causa e consequência. Os participantes de seu teste reportam ter percebido o acender de uma lâmpada anteriormente ao apertar de um botão, mesmo sabendo que a lâmpada se acenderia somente após sua ação.

3 Ver Eagleman \& Pariyadath (2007) para uma importante série de experimentos Oddball em percepção do tempo automático.
}

preciso notar que existem ao menos duas estratégias experimentais. 'Estratégias preditivas' utilizam estímulos propositalmente previsíveis, possibilitando ao cérebro trabalhar à frente da estimulação, considerando os estímulos anteriores como pistas sobre o estímulo seguinte, como nos paradigmas oddball ${ }^{3}$. Já as 'estratégias posditivas' apresentam estímulos imprevisíveis, forçando o cérebro a esperar até que o estímulo seja apresentado. Só então, será possível processar a informação de forma retrospectiva.

Outra distinção relevante, melhor discutida na história do conceito do tempo, é entre duração e ciclo. As durações indicam que um evento se estende por um determinado período. Já os ciclos indicam que um evento poderá se repetir com determinada frequência, em ciclos. O correspondente linguístico seria a distinção entre sentenças durativas como [Durante 4 horas, João trabalhou na restauração], indicando que a duração de um determinado trabalho foi de 4 horas, e sentenças genéricas [Durante 4 semanas, João trabalhou na restauração], indicando que, ao longo de determinado período, alguém trabalhou ciclicamente (ex. todos os dias, independente do tempo diário).

$\mathrm{O}$ artigo de revisão de Buhusi e Meck (2005) organiza um mapa da percepção do tempo no cérebro e identifica três tipos de sistemas, de acordo com a escala de tempo monitorada por cada um deles. O primeiro sistema é o da (i) 'escala dos milissegundos' (milisecond/subsecond timing), também chamado de tempo automático. Devido a sua curta duração, eventos que ocorrem nesta escala são tratados de forma automática pelo cérebro. Esta escala lida basicamente com o controle motor e é processado no cerebelo e nas regiões pré-frontais. A (ii) «escala dos intervalos> (interval timing), varia de alguns milissegundos até aproximadamente 24 horas. Este sistema lida com tomadas de decisão consciente e com a estimação de intervalos/durações de evento, sendo modulado por circuitos córtico-estriados e por neurônios dopaminérgicos. A (iii) 'escala circadiana' (circadian timing), é estudada pela Cronobiologia. O sistema circadiano é responsável pelos ritmos metabólicos, como o de sono-vigília, e se localiza nos núcleos da base cerebral.

Como nosso objetivo é tratar da duração dos eventos linguísticos, este artigo se foca na escala dos intervalos. As pesquisas psicofísicas e neurofisiológicas sobre a percepção dos intervalos de tempo são apoiadas por um modelo de percepção, de aquisição e de recuperação dos intervalos na memória, o Modelo Relógio.

\section{O Modelo Relógio e os intervalos de tempo}

A partir dos dados sobre a percepção e monitoramento do tempo, foi elaborado um modelo psicofísico 
que busca explicar o processamento e prever os resultados de testes comportamentais e neurofisiológicos, especialmente aqueles na escala dos intervalos. O Modelo Relógio (Internal Clock Model ou PacemakerAccumulator Model) foi descrito por Treisman (1963) para resultados comportamentais e foi melhorado ao longo dos anos, até alcançar um modelo que considera os resultados neurofisiológicos em Meck (1996).

$\mathrm{O}$ modelo relógio funciona em três fases: (i) uma fase de acumulação de pulsos, (ii) uma fase de acesso e recuperação das informações das memórias de trabalho e de referência, e (iii) uma fase de comparação/resposta (Fig. 2).

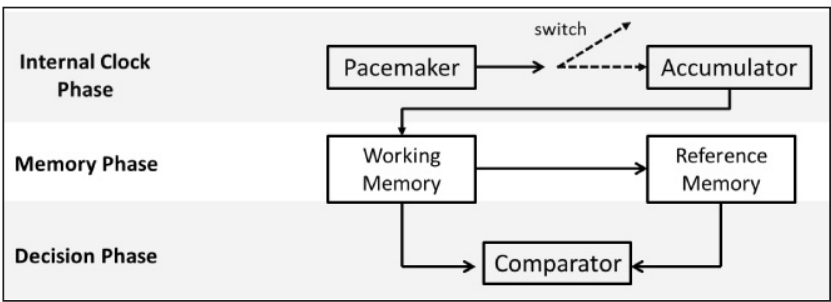

Figura 2. Representação do Modelo Relógio, adaptado de Meck (1996)

Segundo esta proposta, o cérebro emite pulsos ultradianos ${ }^{4}$ numa frequência relativamente estável, como um marcapasso. Esta frequência poderá ser perturbada de acordo com nosso estado físico ou emocional, ou modulado em experimentos farmacológicos, podendo se acelerar ou desacelerar. Quando prestamos atenção em algum evento ou intervalo de tempo, o acumulador é ativado.

Ao final da contagem de pulsos, o output é transmitido para a memória de trabalho, responsável pela estimação da duração. Uma memória de referência será responsável por guardar as informações temporais já experienciadas, construindo um conhecimento sobre determinados eventos/intervalos. Os dois módulos de memória fazem interface com a fase de comparação e de resposta, responsáveis por checar a duração do estímulo ativo na memória de trabalho e por compará-lo com a duração média estimada pela memória de referência.

Sabemos que o cérebro humano realiza uma aquisição estatística de regras e padrões (SAFFRAN et al., 1999). Assim, o modelo propõe que cérebro procura por regularidades na curva estatística das durações dos eventos aos quais somos expostos. Ao recuperar estes eventos na memória, podemos lembrar de um evento

\footnotetext{
4 Ritmos ultradianos são aqueles com frequência baixa, menor que $24 \mathrm{~h}$. No caso do modelo relógio, os pulsos ultradianos teriam frequência na casa dos milissegundos.
}

padrão ou um outlier dependendo do contexto e da precisão da contagem.

Como principais vantagens do modelo, Buhusi e Meck (2005) citam a (i) sua simplicidade e universalidade, permitindo sua utilização em diversas espécies, (ii) a modularidade que separa o relógio dos estágios de memória e de decisão, possibilitando o mapeamento de seus componentes para estruturas cerebrais e para sistemas de neurotransmissores e, finalmente, (iii) o sua simplicidade e seu poder de predição.

Mais além, o modelo relógio também consegue lidar com ilusões temporais. A frequência dos pulsos poderá ser perturbada de acordo com nosso estado físico e emocional, ou modulado em experimentos farmacológicos, podendo se acelerar ou desacelerar. Como o acumulador trabalha com a quantidade de pulsos, estas alterações de frequência resultam em uma medição inexata, reportando para a memória um tempo menor quando a frequência for baixa, ou um tempo maior quando a frequência for alta. Estas modificações na frequência do marcapasso interferem no tempo subjetivo percebido.

\section{Sobre a duração de eventos na linguística teórica e experimental}

A importância dos mecanismos de percepção temporal foi evidenciada em diversos sistemas cognitivos como discutimos anteriormente. Na psicolinguística, fenômenos de percepção temporal foram evidenciados na Percepção da Fala através do Efeito McGurk, do Voice Onset Time e de outros fenômenos motores.

$\mathrm{Na}$ linguística teórica, podemos citar a grande influência do modelo do filósofo Reichenbach (1947), que propõe uma modelo para os tempos verbais baseado em três entidades primitivas: (i) o tempo da fala [S], o tempo do evento [E] e o tempo de referência [R]. Para descrever um tempo verbal, é necessário ordenar estas entidades temporais em uma linha de tempo. Por exemplo, o tempo presente a superposição destes três tempos, ou seja, utilizamos o próprio momento da enunciação como referência para comunicar sobre um evento corrente. Os tempos passado, em geral, se referem a eventos que acontecem antes do momento da enunciação ou do tempo de referência. O contrário aconteceria com o futuro, em que o momento da enunciação precede o tempo de referência. O modelo de gramática de Hornstein (1993) é fundamentalmente baseado no trabalho de Reichenbach.

Como podemos observar, este modelo formaliza os tempos gramaticais, relacionando-os cronologicamente, não estabelecendo relações com a duração de eventos. $\mathrm{Na}$ linguística, este assunto será tratado a partir do conceito de aspecto, em especial, o conceito de 'aspecto lexical', também conhecido como 'aktionsart'. $\mathrm{O}$ aspecto lexical 
indica as propriedades temporais de cada evento. Vale lembrar que, apesar do nome, o aktionsart depende da relação do verbo com outros elementos da sentença, sendo uma propriedade definida de forma composicional.

Um verbo cuja ação é muito rápida é classificado como um verbo de aspecto pontual. Já aqueles que possuem duração perceptível, são classificados como verbos de aspecto durativo. Ambos os tipos de verbos se dividem entre aqueles que possuem um resultado final (pontual: quebrar / durativo: limpar), chamados de verbos 'télicos', e aqueles que não o têm (pontual: espirrar / durativo: correr), conhecidos como verbos 〈atélicos〉. Além destes tipos, é possível observar verbos que denotam um estado atemporal, como ser ou amar, resultando na seguinte classificação de eventos proposta pelo filósofo Zeno Vendler (1967) e expandida por Smith (1991). Esta classificação, porém, é tratada como uma tipologia, não tendo uma interface clara com as ciências cognitivas.

Tabela 1. Classificação de eventos clássica de Vendler expandida por Smith (1991)

\begin{tabular}{lcc}
\hline \multicolumn{1}{c}{ Classe } & Característica & Exemplos \\
\hline Estado & não eventos & $\begin{array}{c}\text { desejar, querer, } \\
\text { amar }\end{array}$ \\
Accomplishment & eventos télicos & pintar, correr \\
durativos & uma milha \\
Achievement & eventos télicos & reconhecer, ganhar, \\
& pontuais & começar \\
Atividade & eventos atélicos & correr, caminhar, \\
& durativos & andar \\
Semelfactivos & eventos atélicos & espirrar, pular, \\
& pontuais & piscar \\
\hline
\end{tabular}

A aproximação da Percepção do Tempo e da Psicolinguística só viria a ser ensaiada nos quatro primeiros testes de Coll-Florit e Genari (2011), em que as autoras propõem uma correlação entre classes de eventos vendlerianas e a percepção da duração subjetiva das ações descritas pelo verbo. Em um de seus testes, os participantes eram apresentados a diversas sentenças do espanhol e deveriam julgar se a sentença fazia sentido ou não. Os estímulos experimentais eram divididos entre sentenças com eventos pontuais (ex. "perder 50 euros") e estados durativos (ex: "deber [dever] 50 euros"). Ao analisar os tempos de resposta, os participantes respondiam mais rapidamente às sentenças com eventos pontuais.

Em um segundo experimento com participantes britânicos, as autoras utilizaram o método de leitura automonitorada com sentenças do inglês flexionadas no imperfeito (ex.: "The doorman | was covering | the sign") ou no perfeito (ex.: "The doorman | covered | the sign") seguidos de uma questão de interpretação. Os resultados apontam para um aumento significativo nos tempos de leitura na região do verbo, podendo se expandir para a região do objeto.

\section{Coerção aspectual}

Um outro fenômeno pode vir a estabelecer uma hipotética interface entre a psicolinguística e a percepção do tempo. Voltando aos exemplos da Tabela 1, note que verbos pontuais são, por definição, incompatíveis com contextos durativos. Apesar disso, é possível compreender perfeitamente uma sentença como (i) [João pulou por dois minutos]. Ao contrário de sentenças durativas como (ii) [João correu por dois minutos], a sentença (i) é interpretada como uma série de eventos (diversos pulos) e não como um evento único (uma corrida). Esta alteração foi batizada com o nome de Coerção Aspectual (PUSTEJOVSKY, 1995; JACKENDOFF, 1997).

No primeiro experimento sobre coerção aspectual, Piñango et al. (1999) tinham dois objetivos. Primeiramente, era necessário saber se a coerção aspectual é real. Em caso afirmativo, seria necessário descobrir se a coerção ocorre no sentido [pontual $>$ iterativo], correspondendo a Hipótese da Coerção Iterativa (ICH) ou no sentido [iterativo $>$ pontual], correspondendo a Hipótese da Coerção Pontual (PCH).

Seu teste consiste na estimulação auditiva de sentenças como as exemplificadas em (1) abaixo. No momento marcado com o (*) uma sequência de letras era apresentada na tela de um computador. A tarefa do voluntário consiste em responder se a sequência de letras correspondia ou não a uma palavra do inglês.

(1) a. The man [examined] the little bundle of fur [for a long time] * to see if it was alive.

b. The man [kicked] the little bundle of fur [for a long time] $*$ to see if it was alive.

Os resultados de Pinãngo e colaboradores apontam um aumento significativo nos tempos de resposta para sentenças como (1b), indicando que (i) a coerção aspectual possui realidade psicológica e (ii) a direção da coerção está de acordo com a hipótese original da ICH. Desta forma, é possível afirmar que sentenças com verbos pontuais em contextos durativos apresentam uma incongruência de ordem aspectual que aumenta o custo de processamento.

Em seguida, Todorova et al. (2000a) testam se estes efeitos se devem a uma coerção ou se o processamento de eventos durativos é mais custoso que o de eventos pontuais. Seu experimento utiliza o método self-paced reading stop making sense e conta com as quatro condições transitivas descritas a seguir. 
(2) a. Even though $\mid$ Howard [sent $\mid$ a large check] $\mid$ to his daughter $\mid$ [for many years], | she refused | to accept his money

b. Even though | Howard $\mid$ [sent $\mid$ large checks] | to his daughter | [for many years], | she refused | to accept $\mid$ his money

c. Even though | Howard [sent | a large check] | to his daughter | [last year], | she refused | to accept his money

d. Even though | Howard [sent | large checks] | to his daughter | [last year], | she refused | to accept his money

As sentenças experimentais foram apresentadas junto a sentenças anômalas e os voluntários foram instruídos a julgar a gramaticalidade dos estímulos. Os resultados apresentam maior rejeição e tempos de resposta significativamente maiores apenas no segmento 5 da condição (2a). Estes resultados evidenciam que (i) a coerção existe e requer um maior custo de processamento; (ii) as propriedades aspectuais provavelmente serão definidas durante a concatenação do verbo com seu objeto direto, uma vez que os verbos podem assumir interpretação iterativa sem apresentar um aumento relevante no custo de processamento se seu objeto for um plural nu como na condição (2b) e; (iii) eventos durativos e eventos iterativos ou com interpretação iterativa não são naturalmente mais custosos que os verbos ou interpretações pontuais.

Segundo estes resultados, coerção iterativa parece ser algum ajuste relacionado à interface sintaxesemântica e parece corroborar hipóteses de interface aspectual como em Tenny (1992), ao mesmo tempo que vai contra os achados mais recentes de Coll-Florit e Genari (2011) de que eventos durativos teriam um maior custo de processamento. Alternativamente, os resultados de Todorova et al. (2000a) podem ser explicados por uma coerção do objeto único e indivisível em (2a). Nesse caso, o ajuste semântico do objeto força o ajuste aspectual do evento.

Os mesmos autores realizaram um segundo teste apresentado em uma conferência no mesmo ano e que não foi publicado (TODOROVA et al., 2000b apud Pickering 2006). Neste experimento, os modificadores temporais durativos [ex.: for many years] foram alterados por modificadores de leitura iterativa [ex.: every year]. Os resultados seguem indicando um aumento significativo no tempo de leitura do segmento 5 mas com uma taxa mais baixa de rejeição das sentenças.

Pickering et al. (2006) então realiza uma série de quatro testes utilizando os mesmos estímulos que Piñango et al. (2006) e Todorova et al. (2000b). Aos estímulos de Piñango et al., os autores inseriram duas versões adicionais em que o modificador temporal aparece em posição pré-verbal como exemplificado no exemplo (1c-d), repetido abaixo.

(1) a. The man [examined] the little bundle of fur [for a long time] * to see if it was alive.

b. The man [kicked] the little bundle of fur [for a long time] $*$ to see if it was alive.

c. [For a long time] the man [examined] the little bundle of fur * to see if it was alive.

d. [For a long time] the man [kicked] the little bundle of fur * to see if it was alive.

Os dois primeiros testes reportados utilizam o método de leitura auto monitorada e uma questão sim/não ao final de cada trial. Nenhum dos dois resultados apresentaram diferenças significativas. Os dois testes seguintes utilizam o método de rastreamento ocular com uma questão sim/ não ao final de cada trial. Os resultados do teste com os estímulos de Piñango et al. não apresentam diferenças significativas. Já no experimento com os estímulos de Todorova et al., os resultados apresentam maiores tempos de fixação na região do objeto singular do que nos objetos plurais com modificadores iterativos. Os autores também reportam um aumento significativo no número de sacadas regressivas na mesma condição.

Brennan \& Pyllkkännen (2008) foram mais cuidadosos com as diferentes interpretações que os verbos podem assumir. Os autores realizaram um pré-teste no qual os participantes deveriam julgar, numa escala de 1 a 7 , o quanto os pares sujeito + verbo (intransitivo) denotam eventos únicos ou eventos múltiplos. Os pares com média menor que 3 foram escolhidos para compor as sentenças experimentais.

Os autores então apresentam dois experimentos: um comportamental de leitura auto monitorada e um neurofisiológico MEG de leitura cinética, ambos com tarefas de julgamento de gramaticalidade. Os estímulos consistiam em sentenças com verbos intransitivos e modificadores temporais pontuais ou durativos no início da sentença, como exemplificado em (3). O julgamento de gramaticalidade não apresentou tempos de resposta significativos. $\mathrm{Na}$ análise online, a região do verbo apresentara um aumento significativo nos tempos de leitura na região do verbo para a condição com coerção, apontando para a hipótese da coerção iterativa.

(3) a. [For many hours] the janitor jumped in the empty hallway

b. [At seven o'clock] the janitor jumped in the empty hallway

No teste MEG, foi utilizado um método de leitura cinética em que cada palavra era apresentada por 
300ms. Os voluntários tinham 4 segundos para julgar a gramaticalidade das sentenças. Os autores encontraram um efeito relativamente similar ao reportado em experimentos sobre a coerção de complemento, apresentando um Anterior Midline Field (AMF) a aproximadamente 400ms no córtex ventromedial pré-frontal direito $(\mathrm{vmPFC})^{5}$.

Outros experimentos também corroboram a hipótese da coerção iterativa utilizando diferentes métodos como rastreamento ocular (TOWNSEND, 2012), leitura auto monitorada (CHAN, 2013) e EEG (PACZYNSKI et al., 2014; YANO, 2016; BŁASZCZAK e KLIMEKJANKOWSKA, a aparecer).

Após quinze anos de experimentação a ICH segue como a hipótese mais aceita na linguística experimental. Por outro lado, esta hipótese voltou a ser desafiada nos últimos anos. Dölling (2014) propõe uma hipótese de coerção baseada em sua classificação de eventos. Em sua proposta, os verbos podem ser separados em diversas classes conforme a forma como eles são utilizados em uma sentença. Uma delas será a interpretação canônica e mais frequente do verbo. A cada utilização, porém, poderá sugerir uma interpretação ligeiramente diferente. Um exemplo é a própria coerção iterativa quando eventos não-resultativos pontuais são utilizados em contextos durativos. O contrário também seria possível quando eventos durativos são utilizados em contextos menores do que o necessário para que possam ser interpretados como eventos completos (ex. "O pianista tocou a sonata por 2 minutos"). $\mathrm{O}$ autor chama esta mudança pelo nome de coerção subtrativa (subtractive coercion).

$\mathrm{Na}$ proposta de Dölling, outras coerções também são possíveis como a 'coerção incoativa' (inchoative coercion), que opera uma mudança de foco de um episódio (ex. João correu por 7 minutos) para um momento (ex. João correu às 7 horas), ou a 'coerção de alongamento' (stretching coercion) que realiza a operação contrária (ex. dormir exatamente às $11 \mathrm{~h} v s$. dormir por uma hora). Ao todo, Dölling (2014) elenca nove tipos de coerção aspectual.

No mesmo ano, Sampaio, França e Maia (2014) propõem outra hipótese, que não se baseia em propriedades linguísticas, mas sim na Percepção do Tempo.

\section{A Percepção do Tempo e o processamento de sentenças}

Sampaio, França e Maia (2014) questionam a coerção aspectual e propõem uma hipótese baseada na própria percepção do tempo. Sua crítica à $\mathrm{ICH}$ se baseia em dois pontos principais. Primeiramente, não parece haver um consenso sobre o conceito de eventos pontuais. Esta noção parece ter origem nas classificações de evento e uma das características mais citadas é a de Moens
(1987, p. 142): “(...) isso não quer dizer que eles [verbos pontuais] deixam de ter uma extensão temporal, mas que sua estrutura interna deixa de ser importante". Isso quer dizer que os eventos pontuais também teriam sua extensão temporal, permitindo que experimentos utilizem verbos como [pular] e [mergulhar] como exemplos deste tipo de verbo. Porém, não podemos dizer o mesmo se observarmos que, nestes verbos, é possível diferenciar claramente o impulso, o salto e a queda.

Uma outra definição bem plausível e empiricamente fundamentada viria, curiosamente, da percepção do tempo:

(...) se um evento durar por menos que alguns milissegundos, ele parece instantâneo - sem duração. Se um evento ou episódio persiste por mais que alguns milissegundos, as pessoas experienciam, lembram e podem então estar aptas a julgar sua duração ${ }^{7}$ (BLOCK, 1990, p. 9 - tradução minha).

Embora o objetivo do autor não seja necessariamente definir eventos pontuais e durativos, me parece perfeitamente possível aplicar tal descrição para fins de descrição linguística.

A segunda crítica é que, caso as noções de eventos pontuais e durativos tenham surgido de fato da classificação de eventos, estes estudos não têm necessariamente o objetivo de explicar como acontece a representação dos eventos, desejando apenas classificá-los. A mesma crítica foi feita por Rosen (1999):

Os estudos em Classificação de Eventos têm o objetivo de identificar um pequeno número de tipos de eventos nos quais todos os eventos possam ser classificados. Esta pesquisa, porém, não é explicativa: ela não indica como os eventos são representados na gramática, nem tenta determinar onde os eventos são codificados - no léxico, na semântica ou na sintaxe. Explicativa ou não, a classificação de eventos indicou características básicas dos eventos que precisam ser representados e estabeleceram um vocabulário para estas características que vem sendo utilizado em virtualmente todas as investigações sobre a representação destes eventos. ${ }^{8}$ (ROSEN, 1999, p. 3 - tradução minha).

\footnotetext{
5 Apesar dos resultados reportados, em conversas pessoais, outros neurocientistas apontam que os efeitos encontrados neste estudo são fracos e não os consideram relevantes.

6 " (...) this does not means that they cease to have a temporal extension, but rather their internal structure is no longer of importance." (MOENS, 1987, p. 142).

7 "If an event lasts for less than a few milliseconds, it seems instantaneous - without duration. If an event or episode persists for longer than a few milliseconds, people experience, remember, and may therefore be able to judge duration." BLOCK (1990, p. 9).

8 "Event Classification research has the goal of identifying a small number of events types into which all events can be classified. This research, however, is not explanatory: it does not address how events are represented in the grammar, nor does it try to determine where events are encoded - within the lexicon, the semantics, or the syntax. Explanatory
} 
Apesar das críticas teóricas, a hipótese apresentada por Sampaio, França e Maia (2014) não contradiz os dados encontrados sobre a coerção iterativa e apenas expande suas previsões, assim como a proposta de Dölling (2014). A contraproposta apresentada pelos autores consiste em estabelecer uma interface entre o modelo relógio e os modelos de processamento linguístico. Para isso seria necessário explicar como acontece a aquisição da informação semântica sobre a duração dos eventos e como ocorre a recuperação desta informação durante o acesso lexical.

No que diz respeito a aquisição das durações, seria possível explicá-la através das duas primeiras fases do modelo relógio. Quando somos expostos pela primeira vez a um evento, o módulo contador acumula a quantidade de pulsos referente ao evento em questão e o guarda na memória de referência. Ao sermos expostos ao mesmo tipo de evento novamente, estas informações vão se acumulando e formando uma curva gaussiana das durações possíveis do evento ${ }^{9}$. Após diversas exposições, temos a capacidade de estimar a duração média e de estabelecer um piso e um teto sobre a duração dos eventos. Excluindo casos especiais que serão outliers, um evento inserido em contextos menores que o piso, seriam considerados eventos incompletos (coerção subtrativa) enquanto aqueles inseridos em contextos que sejam suficientes para que o evento ocorra mais de uma vez, seriam compreendidos como eventos repetidos (coerção iterativa).

Resta explicar como se daria o acesso ao tempo subjetivo no acesso lexical. A hipótese intermodular proposta pelos autores consiste na existência de um contato entre o armazenamento dos itens lexicais dos modelos de linguagem e a memória de referência do modelo relógio. Apenas a título de exemplo, a enciclopédia (lista 3) da Morfologia Distribuída (HALLE, MARANTZ, 1993) faria interface com a memória de referência das durações de evento. Se pensarmos no acesso lexical, no momento em que as informações das palavras são recuperadas pelo processador, as informações linguísticas e temporais sobre o evento seriam relacionadas. Uma hipótese alternativa e mais simples seria indicar que a forma linguística dos eventos conhecidos já é armazenada com suas respectivas durações subjetivas.

Além de descrever os mecanismos através dos quais a cognição de linguagem e de tempo podem interagir no processamento de sentenças, é importante que a

or not, event classification research has pinpointed the basic features of events that needs to be represented, and has established a vocabulary for event characteristics that has been used in virtually all investigation into event representation." (ROSEN, 1999, p. 3).

9 Em uma enquete informal, perguntei a 10 pessoas qual a duração de uma música, sem dar opções de resposta. Todos responderam que músicas duram cerca de 3 minutos. Apesar disso, existem bandas que tocam músicas de mais de 30 minutos, o que seria considerado um outlier. proposta tenha apoio experimental. Como discutido na seção 4, Coll-Florit e Genari (2011) foram os primeiros a evidenciar experimentalmente uma correlação entre duração e classificação de eventos. No que diz respeito aos efeitos de coerção, um experimento simples que compare o padrão de leitura de sentenças com verbos durativos em diferentes contextos temporais poderia evidenciar o acesso às informações temporais durante o processamento online.

Sampaio (2015) apresenta dois experimentos neste sentido, um em Francês e outro em Português do Brasil. Primeiramente foi aplicado um pré-teste para categorizar os verbos em escalas de tempo. Os verbos eram apresentados no centro da tela e os participantes deveriam julgar sua duração entre segundos, minutos, horas e dias. A partir dos verbos com duração na casa dos minutos, foram elaboradas sentenças transitivas como as descritas em (3) abaixo. Todas as sentenças passaram posteriormente por um julgamento de gramaticalidade e de duração de eventos com falantes nativos.

(3) Contextos de Tempo: segundos, minutos, horas e dias Ex.: ${ }_{1}$ Durante $\mid{ }_{2}$ alguns $[\Delta \mathbf{t}] \mid{ }_{3}$ Luara $\mid{ }_{4}$ preencheu $\mid$ ${ }_{5}$ a ficha $\mid{ }_{6}$ na mesa $\left.\right|_{7}$ do escritório

O teste principal utiliza o método de leitura auto monitorada. Os estímulos eram utilizados ora em seu contexto controle [minutos] ora em contextos experimentais [segundos, horas ou dias], segundo um quadrado latino. Os participantes franceses responderam se uma palavra sonda estava presente na sentença anterior. Os participantes brasileiros responderam uma questão $\operatorname{sim} /$ não.

$\mathrm{O}$ experimento em Francês indica um aumento significativo no tempo de leitura para os contextos [segundos] e [horas] no segmento 6, sendo o efeito da condição [segundos] significativamente maior do que na condição [horas]. Já o experimento em português aponta um aumento significativo nas mesmas condições durante a leitura do verbo e do objeto direto, como previsto nos experimentos de coerção aspectual. Uma possível explicação para a diferença nos resultados seria relacionada a diferentes estratégias cognitivas durante a leitura. Uma palavra sonda exige mais memória e um menor compromisso com o sentido da sentença do que uma questão interpretativa.

Repare que a condição [dias], utilizada somente no Português, não apresenta efeitos de coerção. Sampaio, França e Maia (2014) também apresentam um experimento semelhante utilizando as condições [minutos], [dias], [meses] e [anos] em Português. Embora o artigo em questão reporte efeitos de coerção, uma reanálise dos dados em Sampaio (2015) aponta que se 
tratava de falso positivo devido a problemas de análise. Estes resultados parecem indicar que eventos utilizados em durações cíclicas não sofrem coerção. Isso pode indicar que a duração do evento é resolvida já no acesso lexical enquanto eventos habituais são resolvidos em uma fase posterior do processamento linguístico.

\section{Considerações finais}

A pesquisa sobre as hipotéticas influências da percepção do tempo no processamento linguístico ainda é extremamente recente. Apesar disso, as razões teóricas dentro das ciências cognitivas e as evidências psicolinguísticas sobre sua realidade psicológica vêm crescendo a cada ano.

As pesquisas sobre a coerção aspectual, embora busquem por uma explicação 'mais linguística', foram os primeiros trabalhos a tentarem compreender a relação entre linguagem e percepção do tempo. Em um dos primeiros e mais importantes trabalhos sobre o tema, Todorova et al. (2000) afirmam que o custo de processamento de sentenças pontuais e durativas são similares. Essa afirmação é confrontada pelo trabalho de Coll-Florit e Genari (2011). Neste momento, vale lembrar que o experimento de Todorova et al. não parece testar a coerção aspectual em si, mas a uma coerção induzida pelo objeto singular em eventos pontuais resultativos (accomplishments).

Mais recentemente, o trabalho de Sampaio (2015) estuda os eventos cuja duração foi previamente julgada na casa dos minutos. Uma coerção que induza uma leitura repetitiva do evento explicaria o aumento do custo de processamento nas condições experimentais [segundos] e [horas]. Por outro lado, na tarefa de memória, os tempos da condição [segundos] são maiores do que os da condição [horas], o que também confronta os achados de Coll-Florit e Genari. Uma possível explicação para este fenômeno é que processar um evento incompleto é mais custoso do que processar um evento que se repete.

Muitos trabalhos ainda são necessários para que os mecanismos da interface Linguística-Percepção do Tempo sejam descritos de forma satisfatória. Ainda assim, é importante notar que o contato entre a Psicologia e a Linguística, consolidados na década de 60 com o advento da Psicolinguística, seguem encontrando solo fértil para a discussão de ideias comuns para as duas disciplinas e, também, de suas interfaces com a neurociência (SAMPAIO, FRANÇA e MAIA 2015).

\section{Agradecimentos}

Agradeço a Diego Freitas, Filipa Borges, Douglas Bemis, Matthew Matell, Marcus Maia, Aniela França e Virginie van Wassenhove pelas discussões sobre o tema.

\section{Referências}

BŁASZCZAK, Joanna; KLIMEK-JANKOWSKA, Dorota. Aspectual coercion versus blocking: experimental evidence from ERP study on Polish converbs. In: BŁASZCZAK, Joanna (ed.). How categorical are categories? New approaches to the old questions of Tense, Aspect and Mood. Chicago: The University of Chicago Press. (no prelo).

BLOCK, Richard. Cognitive models of psychological time. New Jersey: Erlbaum, Hillsdale. 1990.

BRENNAN, Jonathan; PYLKKÄNEN, Liina. Processing Events: Behavioral and Neuromagnetic Correlates of Aspectual Coercion. Brain and Language, n. 106, p. 132-143, 2008.

BUHUSI, Catalin; MECK, Warren. What makes us tick? Functional and neural mechanisms of interval timing. Nature Reviews Neuroscience, v. 6, p. 755-765, 2005.

CHAN, Ho Leung. Aspectual Coercion in non-native speakers of English. In: KNAUFF, Markus; PAUEN, Michael; SEBANZ, Nathalie; WACHSMUTH Ipke (eds.). Proceedings of the 35th Annual Conference of the Cognitive Science Society, Austin, TX: Cognitive Science Society, 2013. p. 2006-2011.

COLL-FLORIT, Marta; GENNARI, Silvia. Time in language: Event duration in language comprehension. Cognitive Psychology, v. 62, n. 1, p. 41-79, 2011.

DÖLLING, Johannes. Aspectual Coercion and Eventuality Structure. In: ROBERKING, Klaus (ed.). Events, Arguments and Aspects: Topics in the Semantics of Verbs (Studies in Language Companion Series 152). Amsterdan: John Benjamins Publishing, 2014. p. 189-226.

EAGLEMAN, David. Human time perception and its illusions. Current Opinion in Neurobiology, v. 18, n. 2, p. 131-136, 2008.

EAGLEMAN, David; SEJNOWSKI, Terrence. Motion signals bias position judgments: A unified explanation for the flash-lag, flash-drag, flash-jump and Frohlich effects. Journal of Vision, v. 7, n. 4, p. 1-12, 2007.

GEORG, Karsten; LAPPE, Marcus. Spatio-temporal contingency of saccade-induced chronostasis. Experimental Brain Research, v. 180, n. 3, p. 535-539, 2007.

HALLE, Morris; MARANTZ, Alec. Distributed Morphology and the Pieces of Inflection. In: HALE, Kenneth; KEYSER, Samuel Jay (eds.). The View from Building 20. Cambridge: MIT Press, 1993. p. 111-176.

HAGGARD, Patrick. Human volition: towards a neuroscience of will. Nature Reviews in Neuroscience, v. 9, p. 934-946, 2008.

HORNSTEIN, Nobert. As time goes by - Tense and Universal Grammar. Cambridge: MIT Press, 1993.

JACKENDOFF, Ray. The architecture of the language faculty. Cambridge: MIT Press, 1997.

MCGURK, Harry; MACDONALD, John. Hearing lips and seeing voices. Nature, v. 264, n. 5588, p. 746-748, 1976.

MECK, Warren. Neuropharmacology of timing and time perception. Cognitive Brain Research, Elsevier, v. 3, p. $227-$ 242, 1996

MOENS, Marc. Tense, Aspect and Temporal Reference, 1987. 211f. Tese (Doutorado) - University of Edinburgh, Edinburgo, 1987. 
PACZYNSKI, Martin; JACKENDOFF, Ray; KUPERBERG, Gina. When events change their nature: The neurocognitive mechanisms underlying aspectual coercion. Journal of Cognitive Neuroscience, v. 26, n. 9, p. 1905-1917, 2014.

PARIYADATH, Vani; EAGLEMAN, David. The Effect of Predictability on Subjective Duration. PLOS ONE, v. 2, n. 11, p. 1-6, 2007.

PICKERING, Martin; MCELREE, Brian; FRISSON, Steven; CHEN, Lilian; TRAXCLER, Matthew. Aspectual Coercion and underspecification. Discourse Processes, v. 42, p. 131-155, 2006.

PUSTEJOVSKY, James. The generative lexicon. Cambridge: MIT Press, 1995.

REICHENBACH, Hans. (1947). Elements of Symbolic Logic. Reimpresso por Dover Publications, 1980.

ROSEN, Sara Thomas. The syntactic representation of linguistic events, GLOT International, v. 4, n. 2, p. 3-11, 1999.

SAFFRAN, Jenny; JOHSON, Elizabeth; ASLIN, Richard; NEWPORT, Elissa. Statistical Learning of Tone Sequences by Human Infants and Adults, Cognition, v. 70, p. 27-52, 1999.

SAMPAIO, Thiago; FRANÇA, Aniela; MAIA, Marcus. Does Time Perception Influence Language Processing? Self-Paced Reading Evidence of Aspectual Coercion in Durative Events. In: CHRUSZCZEWSKI, Piotr (Org.). Languages in Contact: Ways to Protolanguage 3. Wroclaw: Wyższa Szkoła Filologiczna we Wrocławiu \& Polska Akademia Nauk, 2014. p. 139-156.

SAMPAIO, Thiago; FRANÇA, Aniela; MAIA, Marcus. Linguística, Psicologia e Neurociência: A união inescapável destas três disciplinas, Revista Linguística (UFRJ), v. 11, n. 1, p. 230-251, 2015.

SAMPAIO, Thiago. Coerção Aspectual: uma abordagem linguística da Percepção do Tempo. 2015. 398f. Tese (Doutorado em Linguística) - Universidade Federal do Rio de Janeiro, Rio de Janeiro, 2015.

SMITH, Carlota. The Parameter of Aspect. Dordrecht: Kluwer Academic Publishers, 1991.

STEIN, Barry (ed.). The New Handbook of Multisensory Processing. MIT Press, 2012.

STETSON, Chess; CUI, Xu; MONTAGUE, Read; EAGLEMAN, David. Motor-sensory recalibration leads to an illusory reversal of action and sensation. Neuron, v. 51, p.651-659, 2006.
TENNY, Carol Lee. The Aspectual Interface Hypothesis. In SAG, Ivan; SZABOLCSI, Anna (eds.). Lexical Matters, Stanford: Center for the Study of Language and Information, 1992. p. 1-28.

TODOROVA, Marina; STRAUB, Kathy; BADECKER, William; FRANK, Robert. Aspectual coercion and the online computation of sentential aspect. Proceedings of the 22nd Annual Conference of the Cognitive Science Society, 2000a. p. 3-8.

TODOROVA, Marina; STRAUB, Kathy; BADECKER, William; FRANK, Robert. Processing correlates of aspectual coercion. Comunicação apresentada no Workshop on Events and Paths, ESSLLI XII, Birmingham, England, 2000b.

TOWNSEND, David. Aspectual Coercion in Eye Movements. Journal of Psycholinguitic Research, v. 42, n. 3, p. 281-306, 2012.

TREISMAN, Michael. Temporal discrimination and the indifference interval: Implications for a model of the 'internal clock'. Psychological Monographs, v. 77, n. 13, 1-31, 1963.

URBANTSCHITSCH, Viktor. "Über den Einfluss einer Sinneerregung auf die übrigen Sinnesempfindungen". Pflügers Archiv. European Journal of Physiology, n. 42, p. 154-182, 1888 .

WASSENHOVE, Virginie van; BUONOMANO, Dean; SHIMOJO, Shinsuke; SHAMS, Ladan. Distortions of subjective time perception within and across senses. PLOS ONE, v. 3, n. 1, p. 1-13, 2008

VENDLER, Zeno. Linguistics in Philosophy. Ithaca: Cornell University Press. 1967.

YANO, Masataka. Temporal Dynamics of Syntactic and Semantic Prediction. 2016. 176f. Tese (Doutorado em Linguística) - Kyushu University, Kyushu, Japão, 2016.

ZHAO, Ke; CHEN, Yu-Hsin; YAN, Wen-Jing; FU, Xiaolan. To Bind or Not to Bind? Different Temporal Binding Effects from Voluntary Pressing and Releasing Actions. PLOS ONE, v. 8, n. 5, p. 1-8, 2013.

Recebido: 28 de outubro de 2015 .

Aprovado: 13 de janeiro de 2016.

Contato:mottakun@gmail.com 